\title{
The reference genome of the halophytic plant Eutrema salsugineum
}

\section{Ruolin Yang ${ }^{1+}$, David E. Jarvis ${ }^{1 \dagger}$, Hao Chen ${ }^{1}$, Mark A. Beilstein ${ }^{1}$, Jane Grimwood ${ }^{2,3}$, Jerry Jenkins ${ }^{2,3}$, ShengQiang Shu ${ }^{2}$, Simon Prochnik ${ }^{2}$, Mingming Xin ${ }^{1}$, Chuang $\mathrm{Ma}^{1}$, Jeremy Schmutz ${ }^{2,3}$, Rod A. Wing $^{1}$, Thomas Mitchell-Olds ${ }^{4}$, Karen S. Schumaker ${ }^{1 *}$ and Xiangfeng Wang ${ }^{1 *}$}

1 School of Plant Sciences, University of Arizona, Tucson, AZ, USA

${ }^{2}$ Department of Energy Joint Genome Institute, Walnut Creek, CA, USA

${ }^{3}$ HudsonAlpha Institute of Biotechnology, Huntsville, AL, USA

${ }^{4}$ Department of Biology, Duke University, Durham, NC, USA

\section{Edited by:}

Jun Yu, Beijing Institute of Genomics, China

\section{Reviewed by:}

Jun Yu, Beijing Institute of Genomics, China

Jingfa Xiao, Beijing institute of Genomics, China

*Correspondence:

Karen S. Schumaker and Xiangfeng

Wang, School of Plant Sciences,

University of Arizona, 303 Forbes Hall, 1140 E. South Campus Drive, Tucson, AZ 85721-0036, USA.

e-mail: schumake@ag.arizona.edu; xwang1@cals.arizona.edu

${ }^{\dagger}$ Ruolin Yang and David E. Jarvis have contributed equally to this work.
Halophytes are plants that can naturally tolerate high concentrations of salt in the soil, and their tolerance to salt stress may occur through various evolutionary and molecular mechanisms. Eutrema salsugineum is a halophytic species in the Brassicaceae that can naturally tolerate multiple types of abiotic stresses that typically limit crop productivity, including extreme salinity and cold. It has been widely used as a laboratorial model for stress biology research in plants. Here, we present the reference genome sequence $(241 \mathrm{Mb})$ of $E$. salsugineum at $8 \times$ coverage sequenced using the traditional Sanger sequencing-based approach with comparison to its close relative Arabidopsis thaliana. The E. salsugineum genome contains 26,531 protein-coding genes and $51.4 \%$ of its genome is composed of repetitive sequences that mostly reside in pericentromeric regions. Comparative analyses of the genome structures, protein-coding genes, microRNAs, stress-related pathways, and estimated translation efficiency of proteins between $E$. salsugineum and $A$. thaliana suggest that halophyte adaptation to environmental stresses may occur via a global network adjustment of multiple regulatory mechanisms. The E. salsugineum genome provides a resource to identify naturally occurring genetic alterations contributing to the adaptation of halophytic plants to salinity and that might be bioengineered in related crop species.

Keywords: whole-genome sequencing, genome annotation, Brassicaceae, Eutrema salsugineum, Arabidopsis thaliana, halophyte

\section{INTRODUCTION}

One of the most urgent challenges is increasing agricultural productivity to feed the world's growing population (Godfray et al., 2010). To meet the rising demand for plant-based agricultural commodities, enhanced productivity on land currently in cultivation and expansion into marginal land for agricultural use will be required (Godfray et al., 2010). In either case, a major problem facing agriculture is increased salinity in the soil solution. Salinization affects land that receives little rain and is a growing problem for irrigation agriculture because long-term irrigation leads to the accumulation of salt, resulting in plant salt stress (Khan, 1982). Elevated salt in the soil can cause ionic, osmotic, and oxidative stresses that reduce the yield of important crop plants. According to a recent report from the Food and Agriculture Organization, $\sim 20 \%$ of agricultural land worldwide is affected by soil salinization, causing the loss of arable land at a rate of $1 \%$ per year. Practical solutions for sustainable agricultural outcomes require a multifaceted approach that includes the identification of genetic determinants underlying adaptation to salinity in naturally salttolerant species (halophytes), and the application of this knowledge to enhance salt tolerance in agriculturally and economically important plants.
Eutrema salsugineum (formerly Thellungiella halophila) is native to the seashore saline soils of eastern China and is widely used as a halophytic model for stress-tolerance research in plants (Amtmann et al., 2005; Gong et al., 2005; Amtmann, 2009). E. salsugineum is closely related to the model species $A$. thaliana and the agronomically important Brassica species (e.g., Oilseed rape). The availability of whole-genome sequences of several species in Brassicaceae has opened a new era of comparative genomics in Brassicaceae for a better understanding of genome evolution of this plant family (Dassanayake et al., 2011; Hu et al., 2011; Wang et al., 2011; Cheng et al., 2012; Fang et al., 2012; Hatje and Kollmar, 2012; Tang and Lyons, 2012; Wu et al., 2012; Yang et al., 2012). Because of these relationships, many of the genetic and genomic tools available for $A$. thaliana can be used to facilitate functional and comparative studies of the two species. Previous studies indicate that salt tolerance in E. salsugineum is not derived from complex morphological adaptations, but rather from the enhancement of mechanisms already present in salt-sensitive species (glycophytes) (Bressan et al., 2001; Amtmann, 2009). For instance, reduction in the expression of a single gene in E. salsugineum orthologous to Salt-Overly Sensitive 1 (SOS1) in A. thaliana can dramatically reduce its tolerance to salt (Oh et al., 2009). The similarity of underlying mechanisms increases the likelihood that 
naturally occurring genetic alterations contributing to the adaptation of E. salsugineum to salinity can be bioengineered in related crop species.

Adaptation of plants to environmental stresses can occur through multiple evolutionary and molecular mechanisms. Many studies currently focus on the evolution of gene sequences by detecting amino-acid substitutions subject to positive selection (Wright and Gaut, 2005). In addition, copy number variation arising via gene duplication events may also contribute to stresstolerance, for example, by altering expression dosage of genes in response to stress or creating novel, beneficial functions and/or phenotypes under directional selection (Innan and Kondrashov, 2010). Adaptation can also occur through changes in gene regulation. Transcriptomic comparisons of A. thaliana and E. salsugineum using microarrays and expressed sequence tags (ESTs) revealed that the expression levels of many stress-related genes in E. salsugineum are constitutively higher than their orthologs in A. thaliana, suggesting the transcriptional network is also modulated for a better fitness (Taji et al., 2004, 2010; Wong et al., 2006). Adaption of salt tolerance in plants may also involve the changes in epigenetic regulation systems, such as genes related to DNA methylation pathways have been shown to play important roles in stress responses (Dorantes-Acosta et al., 2012). In addition, it has been reported that changes of translation efficiency of proteins may play important roles in the phenotypic divergence among species (Man and Pilpel, 2007; Jiang et al., 2008). In the current study, we generated the $241 \mathrm{Mb}$ highquality E. salsugineum genome sequence assembled at $8 \mathrm{x}$ coverage and compared it with Arabidopsis thaliana in terms of genome structures, protein-coding genes, microRNAs (miRNAs), stress-related pathways, and estimated translation efficiency of proteins.

\section{MATERIALS AND METHODS ESTIMATION OF THE DIVERGENCE TIME OF E. SALSUGINEUM AND A. THALIANA}

To assess the relationships among published genomes in the Brassicaceae and the E. salsugineum genome, we inferred a phylogeny for 119 species in the family from chloroplastid $(n d h F)$ and nuclear (PHYA) markers. Maximum likelihood phylogeny was inferred in RAxML using the GTRGAMMA algorithm. Subsequently, we combined the markers into a single data set, inferred the phylogeny, and assessed statistical support using the bootstrap method. Finally, we used the dating analysis of Beilstein et al. (2010), which was generated from the same two markers, to estimate the divergence times among the published genomes and $E$. salsugineum.

\section{PREPARATION OF PLANT MATERIALS, DNA, AND RNA EXTRACTION}

Seeds of Eutrema salsugineum (Pall.) Al-Shehbaz and Warwick, accession Shandong, were originally collected by Ziyi Cao in the Yellow River Delta area near Dongying City, Shandong province, China, at $\sim 37^{\circ} 19^{\prime} \mathrm{N}$ and $118^{\circ} 28^{\prime} \mathrm{E}$, and were subsequently inbred for multiple generations. High molecular weight nuclei were isolated from aerial tissue of 4-week-old soil-grown plants using an established protocol (Luo and Wing, 2003), and DNA was extracted using the Qiagen Genomic-tip System, modified for use with plant tissue ${ }^{1}$. Five RNA libraries (CFGU, CFGW, CFHG, $\mathrm{CFHH}, \mathrm{CFHI}$ ) were generated from the following tissues for sequencing (all grown at $21^{\circ} \mathrm{C}$ under a light regime of $16 \mathrm{~h}$ light and $8 \mathrm{~h}$ dark): (1) 17-day-old whole seedlings grown on $0.1 \times$ Murashige and Skoog (MS) media (Murashige and Skoog, 1962) and $1 \%$ sucrose; (2) combined roots and shoots of 5-week-old plants grown hydroponically in a nutrient solution (Gibeaut et al., 1997) (modified by removing $\mathrm{Na}_{2} \mathrm{O}_{3} \mathrm{Si}$ ); (3) combined roots and shoots of 5-week-old plants grown hydroponically in a nutrient solution (Gibeaut et al., 1997) (modified by removing $\mathrm{Na}_{2} \mathrm{O}_{3} \mathrm{Si}$ ), after $6 \mathrm{~h}$ of treatment with $225 \mathrm{mMNaCl}$; (4) aerial portions of 4 -week-old plants grown in soil, after $6 \mathrm{~h}$ at $4^{\circ} \mathrm{C}$; (5) flowers of 4 -week-oldplants grown in soil. RNA was isolated using TRIzol ${ }^{2}$.

\section{IDENTIFICATION OF ORTHOLOGS BETWEEN A. THALIANA AND E. SALSUGINEUM}

To identify orthologs between $A$. thaliana and $E$. salsugineum, protein sequences from the two species were compared using blastp, followed by calculating an overall identity between two aligned sequences using the BioPerl module (Bio::Search::Tiling::MapTiling) that merged the multiple aligned fragments from a pair of proteins to form a single alignment. The best-hits were determined with the highest overall identity. The homology distribution of the best-hits between $A$. thaliana, rice, and E. salsugineum showed that the majority of $A$. thaliana and E. salsugineum best-hit pairs peak at $85 \%$ while the majority of $A$. thaliana and rice pairs peak at $35 \%$. The two distributions crossed at $\sim 60 \%$ identity. Thus, we used $30 \%$ identity as a low-homology cutoff and $60 \%$ identity as a high-homology cutoff.

\section{GENOMIC SYNTENY ANALYSIS BETWEEN A. THALIANA AND E. SALSUGINEUM}

We used i-ADHoRe to identify duplication events in each genome and genomic synteny between two genomes (Simillion et al., 2008). Because a genomic segment containing the same group of genes might be duplicated multiple times, the multiply duplicated segments were merged as one multiplicon (Simillion et al., 2008). Visualization of macro-synteny between the two species was performed using Circos plot (Krzywinski et al., 2009).

\section{ASSEMBLY OF THE SEVEN CHROMOSOMES BASED ON THE KARYOTYPE OF E. SALSUGINEUM}

Based on the chromosomal comparative painting (CCP)-derived karyotype of E. salsugineum, we assembled the seven full chromosomes with consideration of the CentO repeats in the scaffolds to determine the scaffold orientations. We developed a program to automatically assign the scaffolds to the 24 ancestral genome blocks using $A$. thaliana orthologs mapped on the 25 scaffolds in E. salsugineum. Specifically, we first built a table of the 24 ancestral blocks, in which each block contains a group of $A$. thaliana genes assigned with a color-label and a letter-label (A to X) (Schranz et al., 2006; Mandakova and Lysak, 2008). Then, the protein sequences of $A$. thaliana genes were mapped to the 25 scaffolds in E. salsugineum using BLAT, followed by selection of the

\footnotetext{
${ }^{1}$ www.qiagen.com

${ }^{2}$ www.invitrogen.com
} 
best aligned locations (Kent, 2002). We developed a Java program to visualize $A$. thaliana genes mapped on the scaffolds displayed with the corresponding block-colors and block-labels. Third, the scaffolds with color-codes and letter-labels were automatically arranged according to the CCP-derived $(n=7)$ karyotype of E. salsugineum (Mandakova and Lysak, 2008). Fourth, the scaffolds were connected as complete chromosomes. In case the observed discrepancies represent true lineage-specific genome rearrangement, we did not intentionally break any scaffold. Finally, we applied this workflow to the fully assembled genomes of A. lyrata, A. thaliana, E. salsugineum, and $S$. parvula to generate the digital karyotypes.

\section{F-BOX GENE SUPERFAMILY ANALYSIS}

We identified F-box genes in the four genomes using HMMER (Eddy, 1998). The total of 1,912 F-box genes in the four species were clustered using OrthoMCL (Li et al., 2003). We identified 428 groups in which each group contains at least two F-box genes. To identify the lineage-specific F-box genes, we counted the numbers of F-box genes that originated from each species in each group. For instance in Figure 6B, the first group contains $11 \mathrm{~F}$ box genes in E. salsugineum and zero homologous genes in the other three species, representing a family of highly lineage-specific F-box genes in E. salsugineum. To gain a global picture of the evolutionary pattern of F-box genes in the four species, the full-length F-box protein sequences and the $\sim 60$ aa F-box domain sequences were separately aligned using ClustalX (Thompson et al., 1997). Neighbor-joining tree plain files were fed to the APE package to draw the phylogenetic tree (Saitou and Nei, 1987). We computed the proportion of tandemly duplicated F-box genes among the whole F-box superfamily in each species based on the criterion that tandemly duplicated pairs of two adjacent F-box genes were located less than 20 genes apart (Xu et al., 2009).

\section{RESULTS AND DISCUSSION PHYLOGENETIC POSITION OF E. SALSUGINEUM IN THE BRASSICACEAE}

To infer the phylogenetic relationship of E. salsugineum with three other published genomes from the Brassicaceae, including A. thaliana, A. lyrata, and Schrenkiella parvula, we constructed a phylogenetic tree of 119 species in the family from chloroplastid $(n d h F)$ and nuclear (PHYA) markers that have been previously used to provide resolution in the group (Beilstein et al., 2008). The topologies resulting from the analysis of each marker were largely in agreement and, with respect to the placement of species with published genomes, the trees were fully congruent (Figure S1 in Supplementary Material). Thus, we combined the markers into a single data set, inferred phylogeny, and assessed statistical support using the bootstrap method. Finally, we used dating analyses to predict the ages of splits among the published genomes and $E$. salsugineum (Beilstein et al., 2010). Our analyses indicate that E. salsugineum and S. parvula diverged from each other $\sim 38.4$ million years ago (MYA), and that both genomes shared a common ancestor with $A$. thaliana and A. lyrata $\sim 43.2$ MYA (Figure 1).

The divergence time (43.2 MYA) between E. salsugineum and A. thaliana we estimated is much bigger than a previous estimate of 7-12 MYA based on Wu et al. (2012) analysis. Divergent dates between the two studies likely differ due to the use of alternative

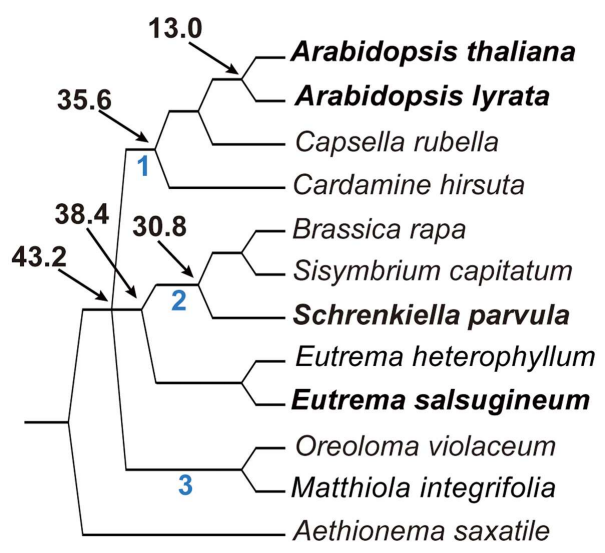

FIGURE 1 | Abbreviated phylogeny of the Brassicaceae inferred from $n d h F$ and $P H Y A$ sequence data shows the relationship and divergence times of the four species.

methodologies and density of taxon sampling. Results of phylogenetic analyses and molecular dating are known to be sensitive to both taxon sampling as well as the number and reliability of fossil calibration points used to date nodes in the inferred tree. Increased taxon sampling and the use of multiple fossil calibrations increase reliability in simulation studies of molecular dating. $\mathrm{Wu}$ et al. inferred dates using a tree with seven species spanning the monocot-dicot split and calibrated with only secondary calibration points (those inferred from other studies, not actual fossil dates). In contrast, our dates were inferred from a tree with a dense sampling of over 150 species in the order Brassicales (to which Brassicaceae belongs) using four distinct fossil calibrations. Moreover, our results showed that, despite the close relationship implied by historical nomenclature between E. salsugineum and S. parvula (formerly Thellungiella parvula), these two halophytes are much more distantly related than previously suggested. E. salsugineum is unequivocally a member of the genus Eutrema, while $S$. parvula represents the earliest diverging branch of Lineage II, and thus its genome serves as a reference point for the emerging Brassica genomes.

\section{GENOME SEOUENCING AND ASSEMBLY OF E. SALSUGINEUM}

We provide a high-quality genome sequence of E. salsugineum at $8 \times$ coverage using a traditional Sanger sequencing-based wholegenome shotgun approach, which was based on a BAC library (average insertion size $130 \mathrm{~kb}$ ) with read length ranging from 500 to $1000 \mathrm{bp}$. A total of $3,875,327$ sequence reads ( $\sim 8 \times$ coverage) were assembled using a modified version of Arachne (Batzoglou et al., 2002) to form 1,107 scaffolds (4,292 contigs) with a scaffold L50 of $9.2 \mathrm{Mb}$, in total of $245.8 \mathrm{Mb}$ of sequences (Table 1). The scaffolds were screened against bacterial proteins, organelle sequences, and GenBank non-redundant (NR) database to remove potential contamination. Additional scaffolds were removed if they consisted of $>95 \% 24$-mers that occurred four other times in scaffolds larger than $50 \mathrm{~kb}$. To improve assembly contiguity, the 1,107 scaffolds were further joined using a combination of $A$. thaliana synteny and BAC and fosmid pair support. Specifically, 
A. thaliana cDNAs (33,413 total) obtained from Phytozome2 were aligned to the assembly of 1,107 scaffolds using BLAT (Kent, 2002), and the best placement (based on cDNA identity) was selected to position the genes. Scaffolds were broken if they contained a linkage group change coincident with an area of low $\mathrm{BAC} /$ fosmid coverage. A total of seven breaks were made, resulting in 1,114 scaffolds in the broken assembly. A. thaliana cDNAs were placed on the broken scaffolds, and a total of 43 scaffolds (208.6 MB) contained significant cDNA alignments. Broken scaffolds were joined by leveraging $A$. thaliana synteny, combined with sufficient BAC/fosmid paired-end link support. Remaining scaffolds were classified into bins depending on sequence content, followed by classification as unanchored rDNA (101 scaffolds), mitochondrion (2 scaffolds), chloroplast (49 scaffolds), or repetitive ( $>95 \%$ masked with 24-mers that occur more than four times in the genome) (192 scaffolds). We also removed 105 scaffolds that were less than $1 \mathrm{~kb}$ in sequence length. The final release contains 639 scaffolds $(243.1 \mathrm{Mb})$ of the E. salsugineum genome (Table 2). To assess the assembly accuracy, a set of 22 BAC clones were sequenced and aligned to the assembly using BLAT (Kent, 2002). The alignments were of high-quality and the overall error rate in this group of clones is $0.17 \%(4,822 \mathrm{bp}$ discrepant out of a possible $2,787,531 \mathrm{bp}$ ), which might be likely due to sequencing error. Therefore, the E. salsugineum genome presented in this study is of high-quality and approximately twice the size of the $A$. thaliana genome and $\sim 100 \mathrm{Mb}$ bigger than the $S$. parvula genome, another published halophytic plant in Brassicaceae (Dassanayake et al., 2011). Compared with another genome sequence of E. salsugineum [called as Thellungiella salsuginea by Wu et al. (2012)] sequenced by Illumina sequencing technology with an average read size of $54 \mathrm{bp}$, the quality of our Sanger-based genome of $E$. salsugineum is substantially higher. In our E. salsugineum genome

Table 1 | Summary statistics of the output of the whole-genome assembly prior to screening, removal of organelles, and contaminating scaffolds and chromosome-scale pseudomolecule construction.

\begin{tabular}{lccccc}
\hline $\begin{array}{l}\text { Size } \\
\text { (bp) }\end{array}$ & $\begin{array}{l}\text { No of } \\
\text { Scaffolds }\end{array}$ & $\begin{array}{l}\text { No of } \\
\text { Contigs }\end{array}$ & $\begin{array}{l}\text { Scaffold } \\
\text { size }\end{array}$ & Basepairs & $\begin{array}{l}\text { \% Non-gap } \\
\text { basepairs }\end{array}$ \\
\hline $5,000,000$ & 21 & 1,699 & $195,535,853$ & $193,524,991$ & 98.97 \\
$2,500,000$ & 28 & 1,891 & $218,149,215$ & $215,804,235$ & 98.93 \\
$1,000,000$ & 37 & 2,086 & $232,605,192$ & $229,708,972$ & 98.75 \\
500,000 & 42 & 2,146 & $236,141,537$ & $233,187,252$ & 98.75 \\
250,000 & 48 & 2,192 & $238,193,084$ & $234,998,491$ & 98.66 \\
100,000 & 54 & 2,224 & $238,995,661$ & $235,442,776$ & 98.51 \\
50,000 & 58 & 2,255 & $239,276,985$ & $235,673,122$ & 98.49 \\
25,000 & 79 & 2,375 & $240,118,515$ & $236,163,550$ & 98.35 \\
10,000 & 198 & 2,680 & $241,758,756$ & $237,686,842$ & 98.32 \\
5,000 & 516 & 3,354 & $243,995,540$ & $239,753,605$ & 98.26 \\
2,500 & 960 & 4,121 & $245,685,632$ & $241,300,862$ & 98.22 \\
1,000 & 1,003 & 4,186 & $245,762,997$ & $241,369,752$ & 98.21 \\
0 & 1,107 & 4,292 & $245,820,000$ & $241,426,360$ & 98.21 \\
\hline
\end{tabular}

The table shows total contigs and total assembled basepairs for each set of scaffolds greater than the size listed in the left hand column. assembly, the N50 (L50) of contigs is $272 \mathrm{~kb}(256.1 \mathrm{~kb})$ and the $\mathrm{N} 50$ (L50) of scaffolds is $8 \mathrm{Mb}(13.4 \mathrm{Mb})$. The top 25 scaffolds $(237 \mathrm{Mb})$ cover $97.4 \%$ of the E. salsugineum genome. The 35 scaffolds longer than $50 \mathrm{~kb}$ cover $98.3 \%$ of the genome. In contrast, the genome provided by Wu et al. (2012) had a contig $\mathrm{N} 50=3.2 \mathrm{~kb}$ and a scaffold $\mathrm{N} 50=403 \mathrm{~kb}$ (Wu et al., 2012).

\section{EST ASSEMBLY AND GENE ANNOTATION}

To facilitate gene prediction in E. salsugineum, we also constructed five RNA libraries generated from the tissues for sequencing (see Materials and Methods). Completeness of the resulting assembly was assessed using 1,138,639 454 ESTs. The aim of this analysis was to obtain a measure of completeness of the assembly, rather than a comprehensive examination of gene expression abundance. Briefly, ESTs $<200$ bps were removed, along with all duplicate ESTs to avoid bias. The remaining ESTs were placed against the genome using BLAT and the resulting placements were screened for alignments that were $\geq 90 \%$ identity and $\geq 85 \%$ EST coverage. The screened alignments indicate that $98.76 \%$ of available expressed gene loci were included in the assembly.

Gene annotation was accomplished using combinatory homology-based searches, ab initio Genome Scan and FgeneSH prediction, and 454-collected ESTs. Specifically, protein sequences from angiosperm plants and the 61,797 EST sequences in E. salsugineum were aligned to the scaffolds to determine the potential coding open reading frames (ORFs). Then, the candidate genomic regions extended by $2 \mathrm{~kb}$ in each direction from the center of aligned ORFs were submitted to Genome Scan and FgeneSH to predict full-length protein-coding genes. The initial $8 \times$ mapped E. salsugineum genome assembly, 454-collected ESTs and the gene annotation can be found at http://www.phytozome.net in the category of Thellungiella halophila (Salt cress). Gene annotation resulted in a total of 26,531 NR protein-coding genes (Table 3 ). Repeat-masking performed on the 639 gap-free scaffolds identified $51.4 \%$ of the genome as repetitive sequences. Orthologs between E. salsugineum and A. thaliana were identified by proteinlevel comparison, with rice genes as contrast to determine the

Table 2 | Final summary assembly statistics for chromosome-scale assembly.

\begin{tabular}{ll}
\hline Scaffold total & 639 \\
Contig total & 3,511 \\
Scaffold sequence total & $243.1 \mathrm{Mb}$ \\
Contig sequence total & $238.5 \mathrm{Mb}(1.9 \%$ gap) \\
Scaffold N/L50 & $8 / 13.4 \mathrm{Mb}$ \\
Contig N/L50 & $272 / 251.6 \mathrm{~kb}$
\end{tabular}

Table 3 | Statistics of predicted genes in E. salsugineum.

\begin{tabular}{ll}
\hline Genome size & $243.1 \mathrm{Mb}$ \\
Total genes & 26,351 \\
Total exons & $137,652 \mathrm{bp}$ \\
Average CDS length & $1,224 \mathrm{bp}$ \\
Average intron length & $363 \mathrm{bp}$ \\
Average gene length & $2,209 \mathrm{bp}$
\end{tabular}


homology cutoff (Figure 2A). Two distinct peaks at $\sim 85 \%$ and $\sim 35 \%$ identity were observed from the homology distributions of E. salsugineum and rice in comparison to A. thaliana, respectively (Figure 2A). Thus, 30 and $60 \%$ overall amino acid identity were used as low- and high-homology cutoffs, respectively. Over $80 \%$ of E. salsugineum genes have high-homology orthologs in A. thaliana (Figure 2B). We used i-ADHoRe (Simillion et al., 2008) to infer genomic synteny between E. salsugineum and A. thaliana based on low-homology orthologs, and identified a similar amount of segmental duplications and syntenic regions, showing that nearly $77.2 \%$ of the E. salsugineum genome is in synteny with $80.2 \%$ of the A. thaliana genome, covering $87.7 \%$ of genes in the E. salsugineum genome and $82 \%$ of genes in A. thaliana (Table 4 ).

\section{HIGH REPEAT CONTENT IN THE E. SALSUGINEUM GENOME}

Visualization of the macro-synteny between the top 25 scaffolds in E. salsugineum (S1-S25) and the five chromosomes in A. thaliana (AtChr1-5) revealed uneven distributions of genes and repeats across certain scaffolds (Figure 3A and Figure S2 in Supplementary Material). To infer the scaffold orientations, we searched for centromere-specific CentO satellite tandem repeats that were used to determine the centromeric ends of scaffolds (Lee et al., 2006). A 177-bp motif (EsCentO) was found tandemly repeated over 500,000 times in E. salsugineum, sharing a similar AT-rich pattern with the 178-bp CentO repeats in A. thaliana (Kumekawa et al., 2000, 2001) (Figure S3 in Supplementary Material). The EsCentOs are abundant among the short scaffolds below $10 \mathrm{~kb}$ and often located at one terminus of a large scaffold, indicating de novo genome assembly was mostly interrupted at the edges of core centromeres (Figure S3 in Supplementary Material). To further examine if the highly repetitive sequences in the scaffolds may form continuous heterochromatin or just were dispersed in the chromosomes, we compared scaffolds 5, 23, 7, 8, and 9 with their syntenic chromosomes in A. thaliana (AtChr). While S5, S23, and S7 consecutively linked as one piece of $31.5 \mathrm{Mb}$ sequence corresponded to the first $20 \mathrm{Mb}$ of AtChr1, S8 and S9 in total of $26.3 \mathrm{Mb}$ corresponded to the other $10 \mathrm{Mb}$ of AtChr1 (Figure 3B). Scaffolds S3 and S1 were inversely arranged in synteny with the short-arm and long-arm of AtChr4, respectively (Figure 3C). While S3 consists of $3 \mathrm{Mb}$ gene-rich and $15 \mathrm{Mb}$ repeat-rich regions, in $\mathrm{S} 1$ a 10 -Mbrepeat-rich half is followed by a $12 \mathrm{Mb}$ gene-rich half (Figure 3C). As multiple heterochromatic knobs adjacent to centromeres were observed in the CCP-derived karyotype of E. salsugineum, we speculated that the $25 \mathrm{Mb}$ repeatrich sequence might correspond to pericentromeric heterochromatin in the E. salsugineum karyotype (Mandakova and Lysak, 2008).

Genome size evolution in plants is highly correlated with the activity of transposable elements (TEs) (Wright and Agren, 2011). Moreover, because environmental stresses can activate excessive TE transposition that may cause damage of genome integrity by inserting into protein-coding regions, the negative effect of TEs should be more efficiently tolerated by halophytes (salttolerant plants) than glycophytes (salt-sensitive plants) (Wessler,
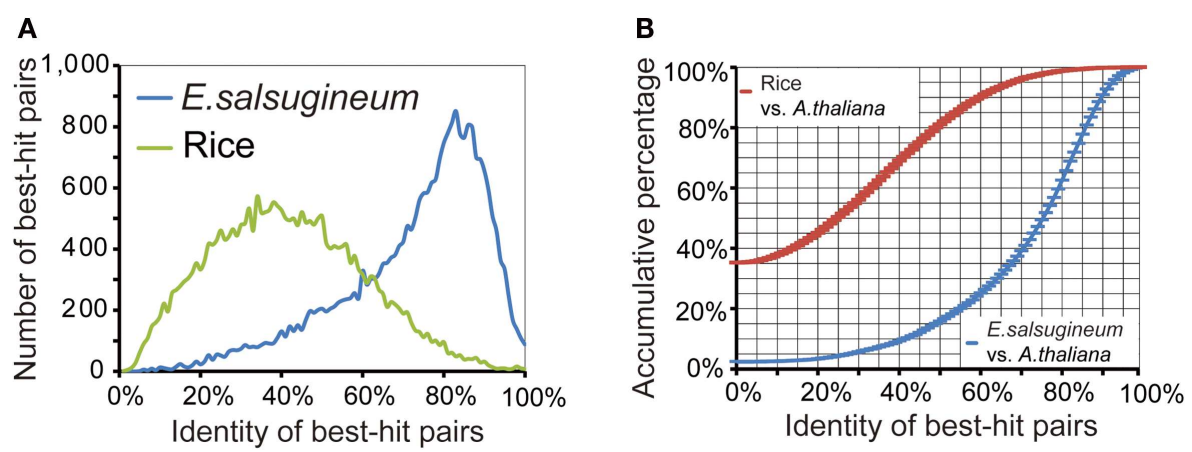

FIGURE 2 | Determination of homology cutoff. (A) Protein-level homology distributions of $E$. salsugineum and rice genes against $A$. thaliana genes. (B) Cumulative percentage of orthologs in E. salsugineum and rice compared to $A$. thaliana.

Table 4 | Identification of duplication events in each genome and genomic synteny between $A$. thaliana and $E$. salsugineum.

\begin{tabular}{|c|c|c|c|c|c|c|}
\hline Within each genome & Multiplicons & Total SDs & Total SD length & $\%$ of genome & Duplicated genes & $\%$ of genes \\
\hline A. thaliana & 200 & 843 & $40.9 \mathrm{Mb}$ & $34.1 \%$ & 10,330 & $35.9 \%$ \\
\hline E. salsugineum & 215 & 1,012 & $91.6 \mathrm{Mb}$ & $37.7 \%$ & 10,067 & $38.2 \%$ \\
\hline Between two genomes & Mulitplicons & Syntenic regions & Synteny length & $\%$ of genome & Syntenic genes & $\%$ of genes \\
\hline A. thaliana & 123 & 2,105 & $96.3 \mathrm{Mb}$ & $80.2 \%$ & 25,232 & $87.7 \%$ \\
\hline
\end{tabular}

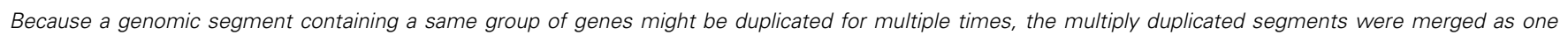
multiplicon (Simillion et al., 2008). 


\section{A}

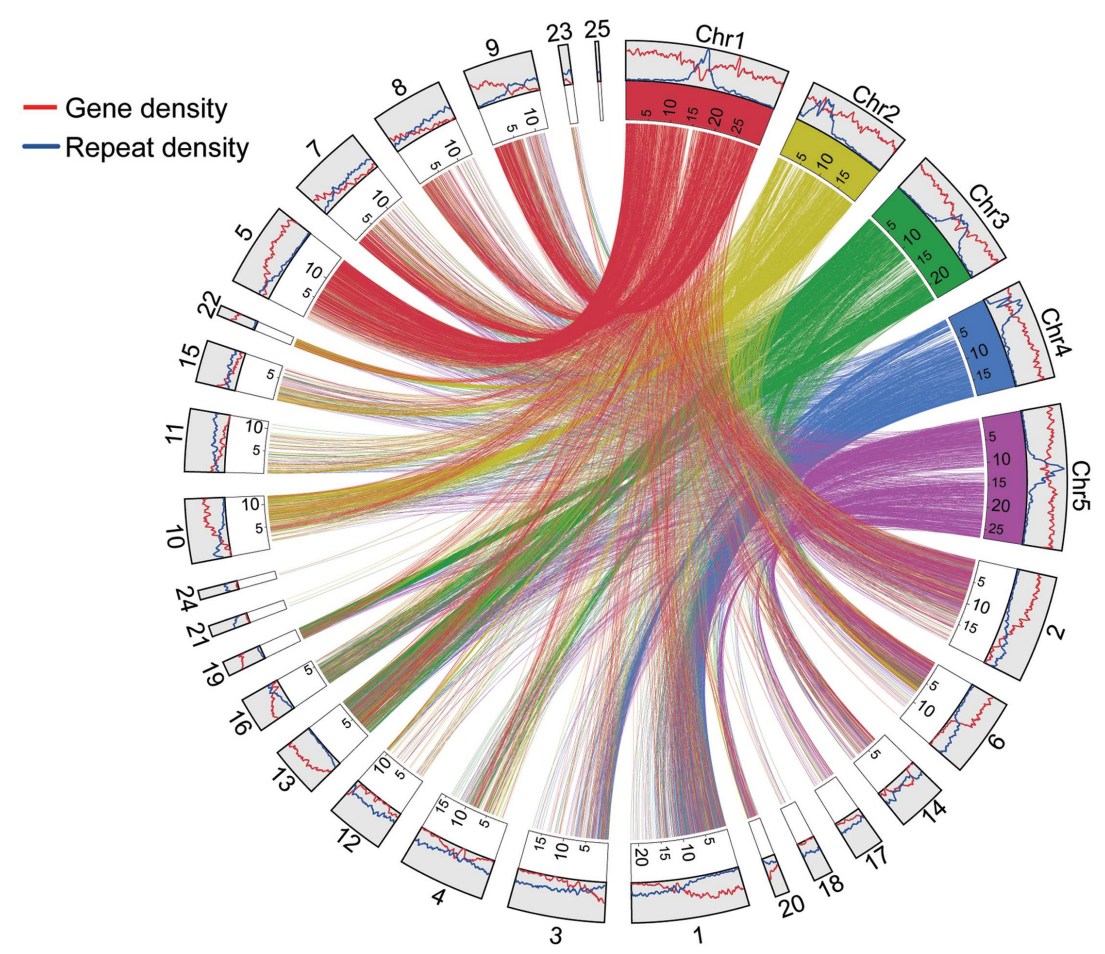

B

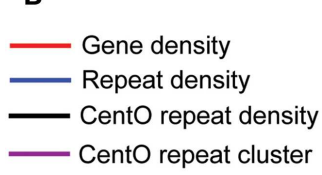

Arabidopsis chromosome $1(\mathrm{Mb})$

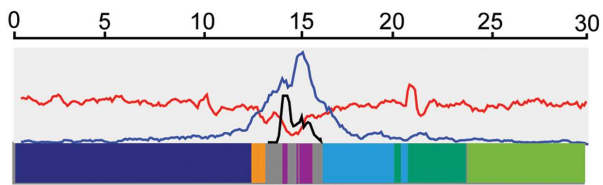

Syntenic regions between Arabidopsis \& Thellungiella

CentO repeat cluster
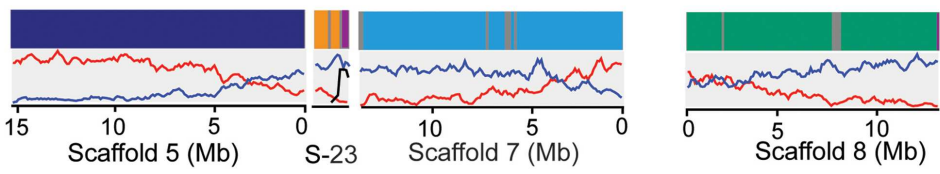

Non-syntenic regions

C
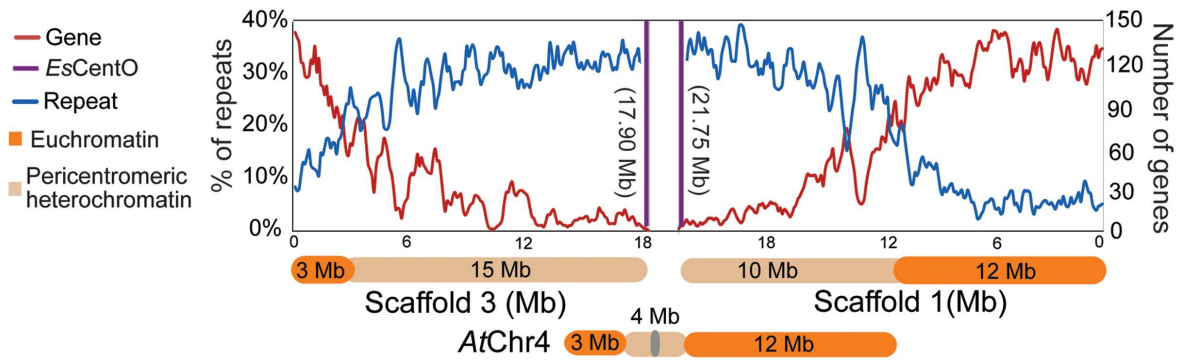

FIGURE 3 | Dramatic expansion of pericentromeric heterochromatin in $\boldsymbol{E}$. salsugineum. (A) Macro-synteny between the 25 largest scaffolds in $E$. salsugineum and the five chromosomes in A. thaliana. (B) Scaffolds 5, 7, 8, 9 are in synteny with At Chr1. (C) Scaffolds S1 and S3 are in synteny with AtChr4.

1996; Fedoroff, 2000). In comparison with the relatively smaller centromere and pericentromere in the A. thaliana genome, the dramatic expansion of pericentromeric heterochromatin in E. salsugineum, which was also reported in Wu et al. (2012) study, may be due to stress-induced activation of TEs. This difference of the genome structure and genome size between E. salsugineum and A. thaliana suggests that the salt-tolerant halophytic species may have evolved particular evolutionary and/or molecular mechanism 
to better tolerate the excessive TE transpositions induced by environmental stress than the salt-sensitive glycophytic species.

\section{DIGITAL KARYOTYPES OF THE FOUR PUBLISHED BRASSICACEAE GENOMES}

Due to the absence of a genetic map for E. salsugineum, assembly of the seven complete chromosomes was based on the $(n=7)$ experimentally derived karyotype of E. salsugineum (see Materials and Methods) (Mandakova and Lysak, 2008). The A. lyrata genome represents the ancestral $(n=8)$ karyotype for much of the Brassicaceae, consisting of 24 conserved chromosomal blocks designated A-X (Schranz et al., 2006; Mandakova and Lysak, 2008). Each block includes a set of $A$. thaliana genes in synteny with their orthologs in other Brassicaceae species (Schranz et al., 2006; Mandakova and Lysak, 2008). We developed a bioinformatic tool KGB assembler to finish the assembly of complete chromosomes of Brassicaceae species from the scaffold sequences according to the experimentally derived karyotypes provided by users (Ma et al., 2012). Specifically, the scaffolds of E. salsugineum were automatically assigned to the 24 ancestral genome blocks based on the order of the A. thaliana genes mapped on the scaffold sequences. Then, the syntenic regions were visualized with the corresponding block-colors and block-labels associated with the A. thaliana genes, followed by manual adjustment of the scaffolds on each chromosome according to the block layouts in the E. salsugineum karyotype. The positions of EsCentO repeats and the density of genes and repeats on the scaffolds were also considered to ensure the correct orientations of scaffolds, since genomic inversions may exist between E. salsugineum and A. thaliana. Based on this analysis, we generated the in silico karyotypes of E. salsugineum, A. thaliana, A. lyrata, and S. parvula to visually inspect the four genome assemblies (Figure 4). The arrangements of the 24 blocks in A. lyrata and A. thaliana are consistent with the CCP-derived karyotypes, except that a $\sim 1 \mathrm{Mb}$ region in the D-block of AlChr2 is located on AlChr1 (Figures 4A,B). In S. parvula, two discrepancies were observed, including a flip of the M-block and N-block on SpChr2 and an inverse order of the V- to X-blocks on SpChr5, which were likely due to assembly errors in the second version of the genome release (Figure 4C). The E. salsugineum karyotype had parts of the M-block on EsChr5 and EsChr3 and parts of H-block on EsChr3 and EsChr4 (Figure 4D). These digital karyotypes provide a starting point to facilitate the improvement of genome assembling in future.

\section{AMPLIFICATION OF THE F-BOX GENE FAMILY IN E. SALSUGINEUM VIA TANDEM DUPLICATION}

Based on the finalized genome assembly, we created a chromosome-scale synteny map to illustrate genomic rearrangements between E. salsugineum and A. thaliana based on the low-homology ortholog pairs (Figure 5). A syntenic block covering a $\sim 5 \mathrm{Mb}$ region on AtChr3 and a $\sim 15 \mathrm{Mb}$ region on $E s \mathrm{Chr} 3$ includes 59 and 78 tandemly arrayed F-box genes in A. thaliana and in E. salsugineum, respectively (Figure 5). Phylogenetic analysis of these 137 F-box genes in the two species revealed that $E$. salsugineum contains more lineage-specific F-box genes derived from tandem duplication (Figure 5). In plants, F-box genes comprise a rapidly evolving superfamily encoding a subunit of the
SCF complex, an E3 ubiquitin ligase catalyzing substrate-specific protein degradation (Xu et al., 2009). To examine whether the F-box gene families are substantially different among the Brassicaceae species, we included the other two Brassicaceae species with complete genome sequences. We identified 613, 505, 602, and 192 F-box genes in E. salsugineum, A. thaliana, A. lyrata, and S. parvula, respectively (Figure 6A). Among the four species, E. salsugineum contains the highest proportion $(\sim 55 \%)$ of tandemly duplicated F-box genes, while $S$. parvula contains the lowest (Figure 6A). To identify lineage-specific F-box genes for each species, we clustered protein sequences of the 1,912 F-box genes using OrthoMCL (Li et al., 2003), and identified 428 groups with a minimum of two F-box genes in one group (Figure 6B). Because the only wellconserved region among all the F-box genes is the F-box domain ( $\sim 60$ amino acids), the high sequence divergence in the remainder of the F-box genes resulted in a high number of clusters of homologous genes. Genes clustered together in the same species may be indicative of lineage-specific F-box gene proliferation. Phylogenetic analysis based on both the complete gene sequences and the F-box domains revealed that E. salsugineum contains the highest number of species-specific F-box genes among the four species (Figure 6C). The numbers of F-box gene super-families have been reported to be highly variable in plants (Xu et al., 2009). While there is no substantial difference in terms of copy number variations of F-box genes between salt-tolerant E. salsugineum and salt-sensitive A. lyrata, the number of F-box genes in salt-tolerant S. parvula is significantly different from E. salsugineum. Thus, whether the different contents of F-box genes and the biological consequence of either amplification or contraction of F-box genes in halophytic plants is correlated with its tolerance to salinity requires further investigation.

\section{SIMILAR COPY NUMBER OF GENE FAMILIES BETWEEN E. SALSUGINEUM AND A. THALIANA}

Gene duplication is recognized as a pivotal driving force for stressadaptive evolution (Hittinger and Carroll, 2007; Deng et al., 2010). Gene copies arising from whole-genome duplication event may be either lost or develop new biological functions, which can be inferred from their differential expression patterns (Fang et al., 2012; Ford et al., 2012). We therefore compared gene copy number in families of transcription factors (TFs) and the families of SOS-like genes in E. salsugineum and A. thaliana. Most TF gene families contain equal or near-equal numbers (Figure S4 in Supplementary Material). Analysis of the SOS-like genes including Cation/H+Exchanger (CHX), Calcineurin B-Like Interacting Protein Kinase (CIPK), Calcium Dependent Protein Kinase (CDPK), Calcineurin B-Like $(C B L)$, and antiporter families revealed that, in most cases, A. thaliana has more copies than E. salsugineum (Figure 7A and Table S1 in Supplementary Material). Moreover, most extra copies of the SOS-like genes in A. thaliana originated via tandem duplication (Figure 7B). When we searched for expanded gene families among all the functional categories, we found that the only class showing statistically higher enrichment in E. salsugineum compared to $A$. thaliana was "ubiquitin-dependent protein modification" (Figure 7C and Figure S5 in Supplementary Material). This result is consistent with the observed amplification of the F-box gene family in E. salsugineum, since the 


\section{A}

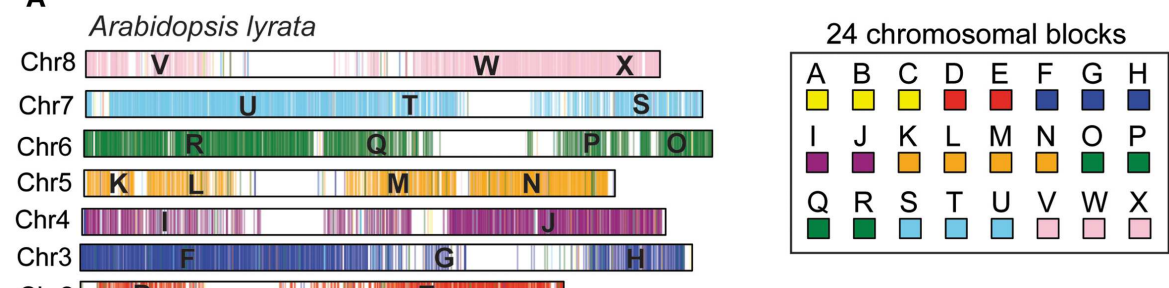

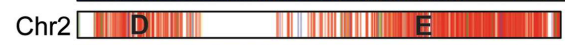

\begin{tabular}{cccc|c|c|c|c|c|c|c|} 
Chr1 & A & & B & & & & C & & \\
\hline 0 & 5 & 10 & 15 & 20 & 25 & 30 & $35(\mathrm{Mb})$
\end{tabular}

B

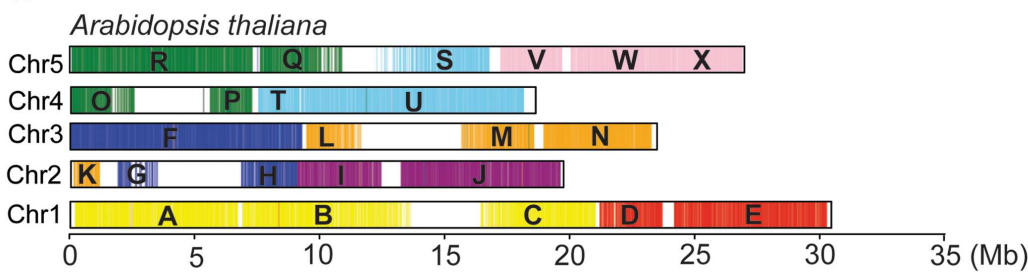

C

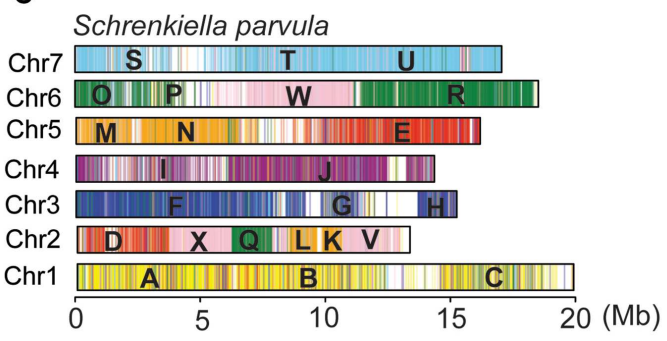

D

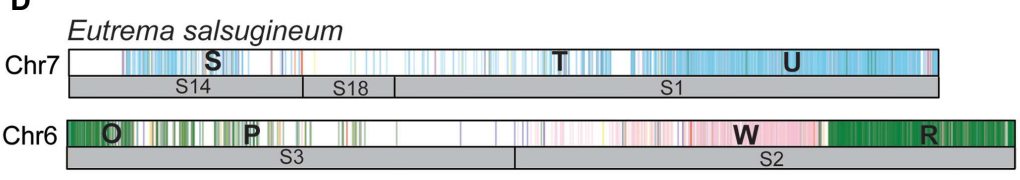

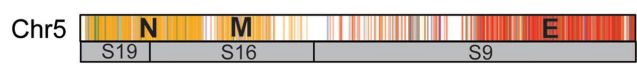

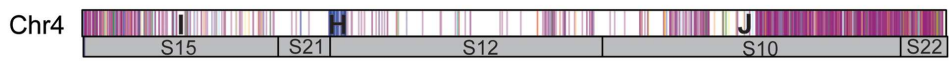

Chr3

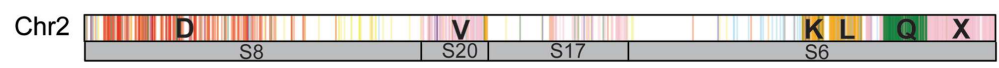

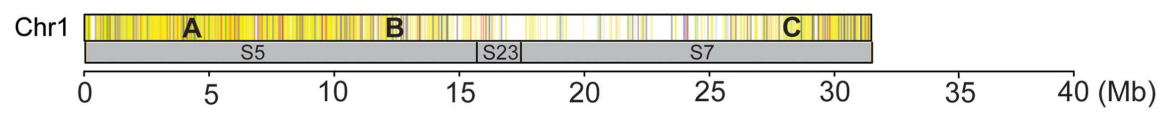

FIGURE 4 |The digital karyotypes of the A. lyrata (A), A. thaliana (B), S. parvula (C), and E. salsugineum (D) genomes.

function of certain subfamilies of F-box genes is to recognize specific substrates in ubiquitin-dependent protein degradation pathways. As certain members of the F-box family are involved in stress-response pathways, functional analyses of the duplicated F-box genes in E. salsugineum will determine whether the lineagespecific proliferation of F-box genes in E. salsugineum contributes to stress-tolerance (Gagne et al., 2002).

\section{STRESS-RELATED MICRORNAs SUBJECT TO TANDEM DUPLICATION}

MicroRNAs have been frequently reported as critical posttranscriptional regulators of stress-response pathways in both plants and animals. To determine whether stress-related miRNA families are also subject to duplication, we computationally predicted the genomic loci encoding miRNAs in the E. salsugineum genome and compared them with $A$. thaliana miRNAs. Because currently there 


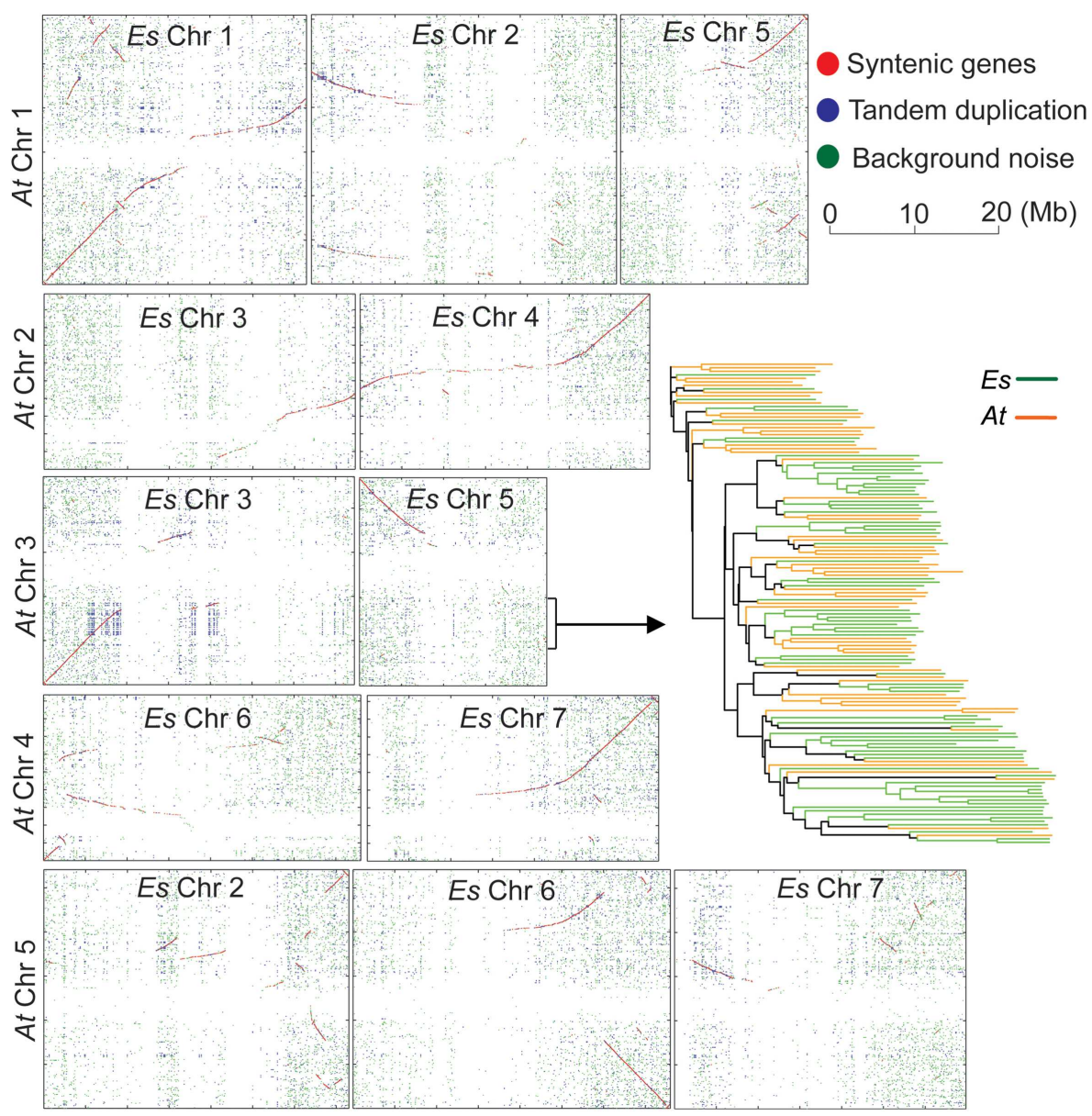

FIGURE 5 | Chromosome-scale synteny between A. thaliana and $\boldsymbol{E}$. salsugineum. Each dot represents a pair of orthologs between $A$. thaliana and $E$. salsugineum. Red dots, macro-synteny orthologs. Blue dots, tandemly duplicated orthologs. Green dots, background noise.

is no available small RNA sequencing data, our computational prediction of miRNA loci in E. salsugineum may only identify the highly conserved miRNA families between E. salsugineum and A. thaliana. First, the mature miRNA sequences in A. thaliana obtained from miRBase were mapped to the genome sequence of E. salsugineum by Bowtie (Langmead et al., 2009). Then the premiRNA hairpins in E. salsugineum were predicted by miRDeep-p, a suite of tools that have been optimized based on the characteristics of pre-miRNA hairpins in plants (Yang and Li, 2011). At last, the predicted pre-miRNA hairpins were manually examined to identify bona fide miRNA genes in E. salsugineum according to the secondary structures of candidate pre-miRNA hairs. We identified 141 high-confidence miRNA-encoding loci in E. salsugineum, corresponding to 151 miRNA loci in A. thaliana (Table S2 in Supplementary Materials). Additionally, seven genomic regions containing A. thaliana mature miRNA sequences in E. salsugineum showed ambiguous hairpin structures. As expected, we found expansion of numerous previously reported stress-related miRNAs in E. salsugineum, with potential gene targets in a wide range of stress-related pathways. For instance, four copies of miR164 (miR164a to miR164d) were identified in E. salsugineum while three copies were found in A. thaliana, which have been reported to post-transcriptionally regulate mRNA transcripts of NAC, a transcription factor required for $\mathrm{ABA}$-independent pathway in response to a variety of abiotic stresses (Kim et al., 2009). Another miRNA family MIR167 targeting genes encoding Auxin response factor (ARF) has six members (MIR167a to MIR167f) in E. salsugineum but four members (MIRR167a to MIR167d) in A. thaliana (Rhoades et al., 2002). A number of miRNA genes showed tandem duplication patterns in E. salsugineum, including MIR168a and MIR399a which were tandemly duplicated four times on EsChr7 and EsChr1, respectively (Figure 8 and Table S2 in Supplementary Material). While miR168a targets ARGONAUTE 1 (AGO1) acting as a negative-feedback mediator to control $A G O 1$ expression, miR399a is stress-inducible and targets $\mathrm{PHO} 2$ transcripts which encode E2 ubiquitin-conjugating enzymes (Lin et al., 2008; Li et al., 2012).

\section{HIGH TRANSLATIONAL EFFICIENCY OF TRANSPORTATION-RELATED GENES IN E. SALSUGINEUM}

To determine whether variation in translational efficiency of messenger RNAs to proteins caused by codon usage bias may 

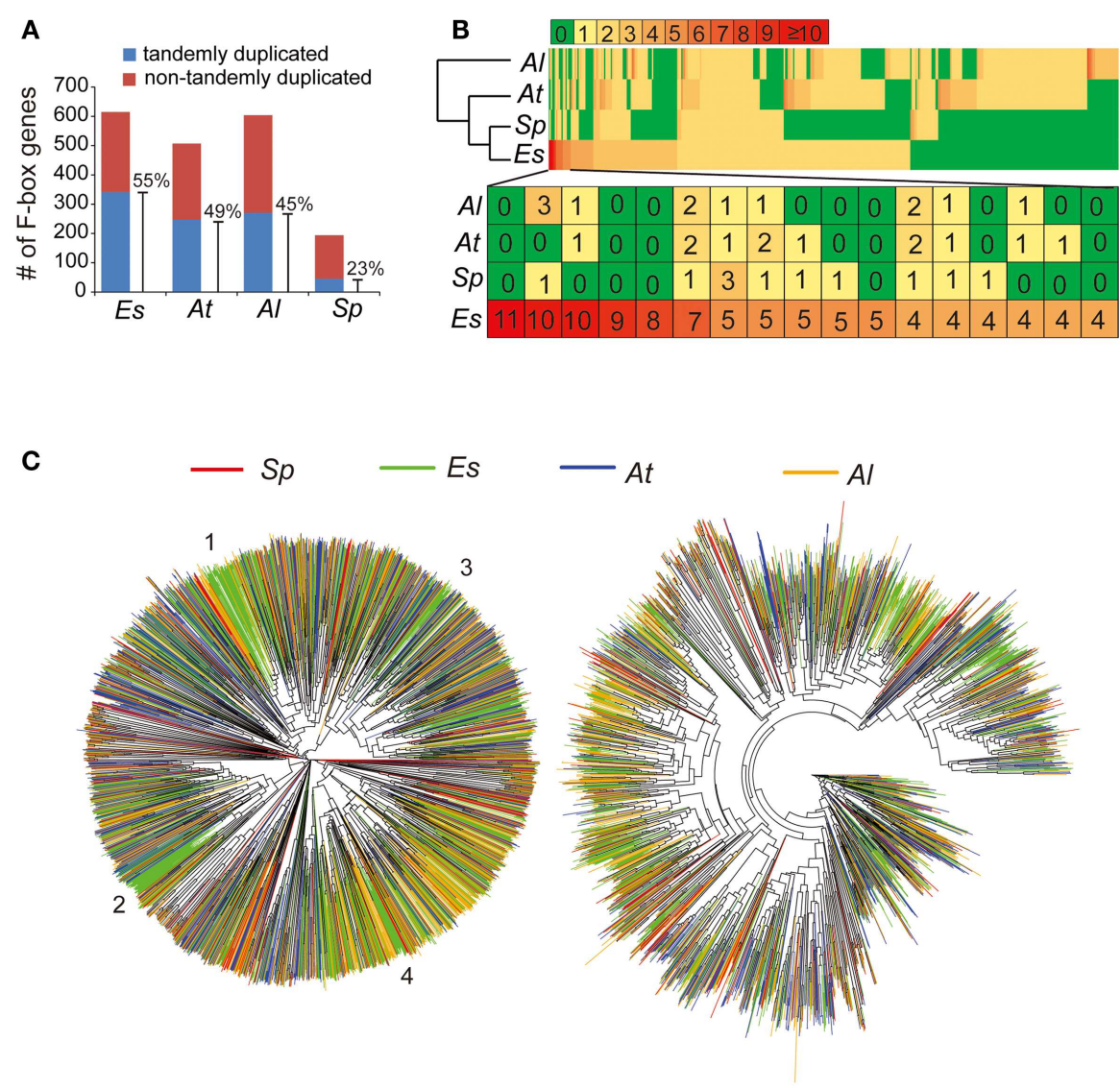

FIGURE 6 | Expansion of F-box gene family in E. salsugineum.

(A) Tandem duplications contribute to F-box gene family expansion. (B) The lineage-specific F-box genes in the four species. A total of 1,912 orthologous F-box genes in the four species were clustered to 428 clusters with at least two F-box genes. (C) Phylogenetic tree analysis of the total of $\sim 1900 \mathrm{~F}$-box genes identified in the four species. The tree on the right was built based on the $\sim 60$-aa F-box domain. Four $E$. salsugineum specific F-box clades were numbered by $1-4$. The tree on the left was built based on the alignment of full CDS sequences of F-box genes in the four species. contribute to the ability of E. salsugineum to tolerate extreme environments, we computed the tRNA adaptation index (tAI) among the gene families in the two species for each orthologous gene using the codon R software (dos Reis et al., 2004). Specifically, the tRNA pool was first obtained by running tRNAs can-SE software in the two genomes (Lowe and Eddy, 1997). Then, based on the classes of tRNAs, codon usage bias for each gene was computed (Figure 9A). Although the codon usage biases (tAIs) of the orthologs between A. thaliana and E. salsugineum were globally similar, we also observed that there were more genes with higher tAT in E. salsugineum than those in A. thaliana, indicating a group of genes in E. salsugineum may be more efficiently translated than their orthologs in A. thaliana (Figure 9A). Subsequently, we performed a Wilcoxon rank-sum test to identify the specific gene families whose members showed significantly increased translation rates in E. salsugineum relative to those in A. thaliana. Our analysis identified 21 gene families showing significantly higher translational rates $(p \geq 0.05)$ in E. salsugineum than in A. thaliana, including a variety of membrane-associated transporter families and certain TFs, such as ABC transporters, antiporters as well as a variety of membrane-associated proteins (Figure 9B and Table S3 in Supplementary Material). However, among the core SOS-like gene families, such as CBL family, CDPK family, and CIPK family, no similar trend was observed (Figure 9B). These results suggest that the translation of proteins related to ion transportation in E. salsugineum may be more efficient than in A. thaliana, which may result in a quick response to stress through biased codon usage.

\section{CONCLUSION}

In this study, we present the whole-genome sequence of $E$. salsugineum and compare it to the published genome of A. thaliana. Our analyses collectively suggest that the stress-tolerance of $E$. salsugineum is unlikely to be determined by variation in a few genes; rather, a global network adjustment of multiple regulatory mechanisms, involving transcriptional, posttranscriptional, translational, and posttranslational systems, may be responsible for its adaptation to extreme environments. As a consequence, a spectrum of changes in life cycle, morphology, cellular components, and molecular pathways result in adaptation of E. salsugineum to environmental stresses (Gong et al., 2005; Wong et al., 2006; Amtmann, 2009; Bouchereau et al., 2010; Oh et al., 2010). 

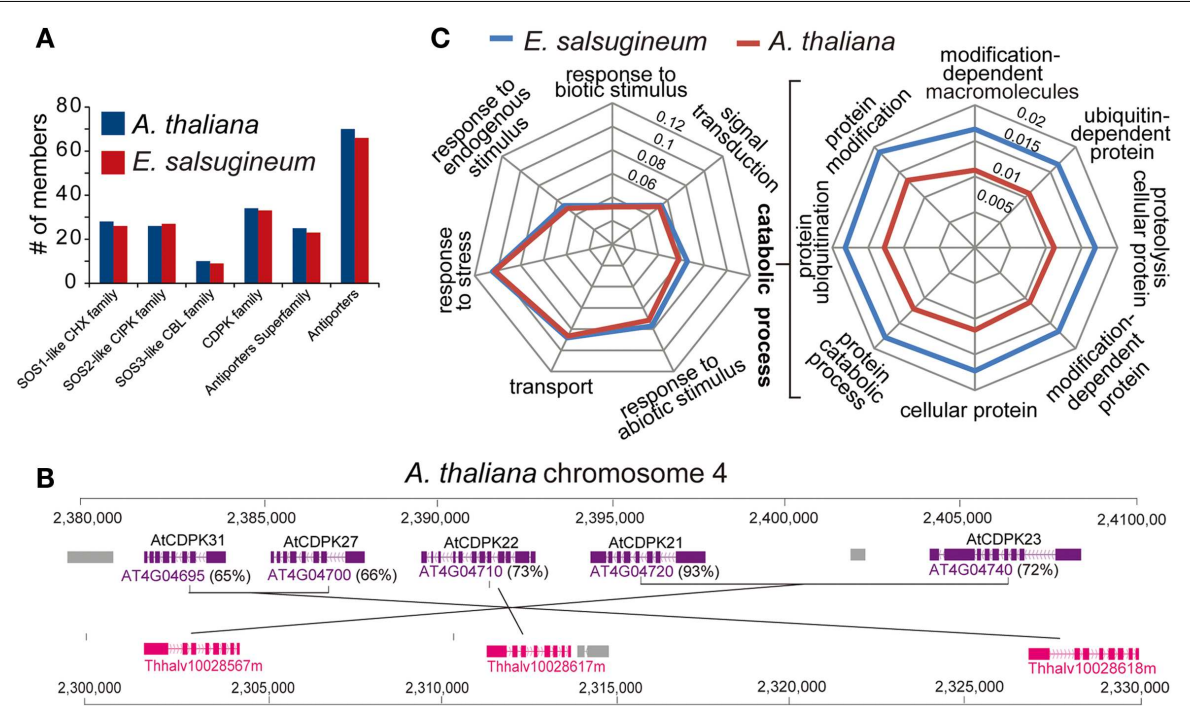

E. salsugineum chromosome 6

FIGURE 7 | (A) The composition of SOS-like gene families is similar in $A$. thaliana and E. salsugineum. (B) A genomic region on AtChr4 shows two tandem duplication events in $A$. thaliana increasing the gene dosage of the AtCDPK family. (C) The genes involved in "ubiquitin-dependent protein modification" are more enriched in E. salsugineum than in $A$. thaliana.

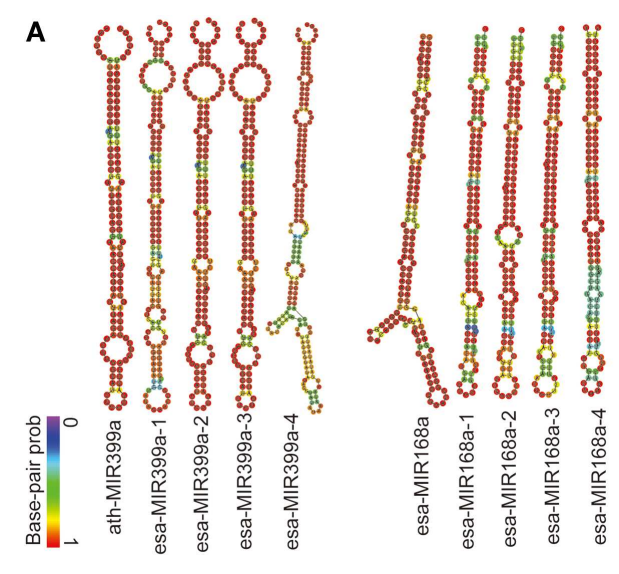

FIGURE 8 | Tandemly duplicated stress-related miRNAs in $E$. salsugineum. (A) The hairpin structures of ath-MIR399a and ath-MIR168a in A. thaliana, and the four copies of esa-MIR399a and esa-MIR168a miRNA

\section{ACCESSION}

This Whole-Genome Shotgun project has been deposited at NCBI GenBank under the accession PRJNA73205. The version described in this paper is the first version. Genome annotation of E. salsugineum is publically available at http://www.phytozome.net in the category of Thellungiella halophila (Salt cress).

\section{AUTHORS CONTRIBUTION}

Karen S. Schumaker, Rod A. Wing, and Thomas Mitchell-Olds conceived the project; David E. Jarvis prepared the plant materials and the samples; Jeremy Schmutz, Jane Grimwood, and Jerry Jenkins sequenced and assembled the genome; Simon Prochnik and ShengQiang Shu annotated the genome. Ruolin Yang, Hao Chen, Xiangfeng Wang, Chuang Ma, Mingming

\section{B}
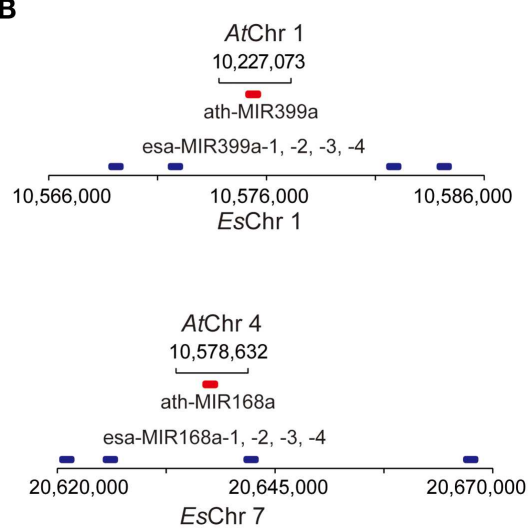

precursors in E. salsugineum. (B) Copy number variation of stress-related microRNA genes MIR399a and MIR168a between A. thaliana and E. salsugineum.

Xin, analyzed the data; and Xiangfeng Wang, Ruolin Yang, Karen S. Schumaker, David E. Jarvis, and Mark A. Beilstein wrote the manuscript with contributions from all authors.

\section{ACKNOWLEDGMENTS}

The genome sequencing, assembly, and annotation conducted by the U.S. Department of Energy Joint Genome Institute is supported by the Office of Science of the U.S. Department of Energy under Contract No. DE-AC02-05CH11231. We thank Kerrie Barry for project management and support at the JGI. Thomas Mitchell-Olds was supported by NIH grant R01GM086496. We are also grateful to Dr. Stephen Wright for helpful comments on the manuscript. 
A

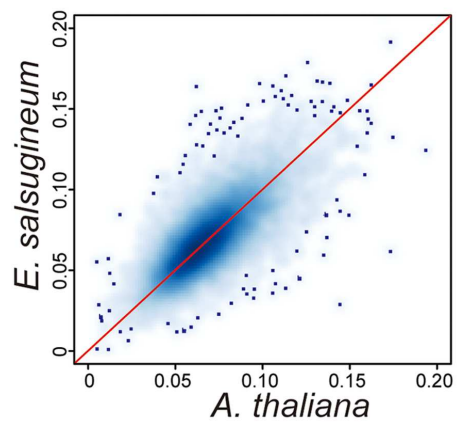

B

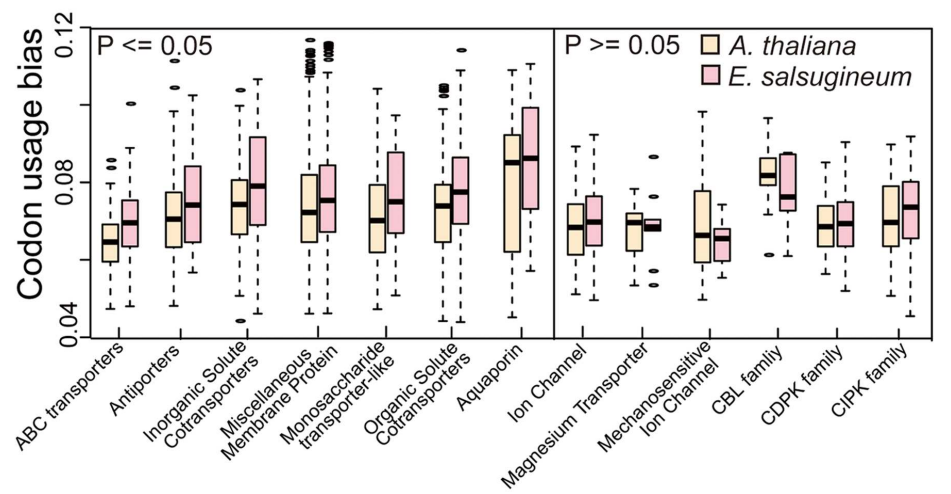

FIGURE 9 | High translational efficiency of transportationrelated genes in E. salsugineum. (A) Kernel density plot showing the globally similar codon usage bias (tAl) between $A$. thaliana and
E. salsugineum. The $x$-and $y$-axis denote the tAl coefficient of genes. (B) Comparison of codon usage bias (tAl) of genes in terms of functional categories.

\section{SUPPLEMENTARY MATERIAL}

The Supplementary Material for this article can be found online at http://www.frontiersin.org/Plant_Genetics_and_Genomics/10. 3389/fpls.2013.00046/abstract

Table S1 | Lists of orthologs of stress-related SOS (Salt-Overly Sensitive) gene families. The genes in red indicate the tandem duplications. The $E$. salsugineum genes marked with asterisks indicates two copies in A. thaliana correspond to one copy in E. salsugineum.

Table S2 | microRNA gene families related to stress-response pathways computationally predicted in E. salsugineum.

Table S3 | Comparison of translational efficiency among the gene families between the $A$. thaliana and $E$. salsugineum.

Figure S1 | Maximum likelihood phylogeny inferred in RAxML 7.2.8 using the GTRGAMMA algorithm. The alignment comprised $n d h F(2016 \mathrm{bp}$ ) and PHYA (1731 bp) sequences for 119 species of Brassicaceae and two outgroups in Cleomaceae. The data were partitioned by gene, and thus each partition was permitted to evolve independently. Numbers above nodes are likelihood bootstrap values from 100 replicates. Highlighted bootstrap values show the distinct placements of Schrenkiella parvula (formerly Eutrema parvulum) and Eutrema salsugineum (formerly Thellungiella halophila).

Figure S2 | Gene densities and repeat densities are unevenly distributed in the top 25 scaffolds. (A) The top 25 scaffolds are sorted in order of their sizes.

\section{REFERENCES}

Amtmann, A. (2009). Learning from evolution: Thellungiella generates new knowledge on essential and critical components of abiotic stress tolerance in plants. Mol. Plant 2, 3-12.

Amtmann, A., Bohnert, H. J., and Bressan, R. A. (2005). Abiotic stress and plant genome evolution. Search for new models. Plant Physiol. 138, 127-130.

Batzoglou, S., Jaffe, D. B., Stanley, K., Butler, J., Gnerre, S., Mauceli, E., et al. (2002). ARACHNE: a wholegenome shotgun assembler. Genome Res. 12, 177-189.

Beilstein, M. A., Al-Shehbaz, I. A., Mathews, S., and Kellogg, E. A. (2008). Brassicaceae phylogeny inferred from phytochrome $a$ and Ndhf sequence data: tribes and trichomes revisited. Am. J. Bot. 95, 1307-1327.

Beilstein, M. A., Nagalingum, N. S., Clements, M. D., Manchester, S. R., and Mathews, S. (2010). Dated molecular phylogenies indicate a

(B) Scaffold 1, 2, 5, 6, 9, 10, 13 have higher numbers of protein-coding genes while scaffold 3, 4, 7, 8, 11, and 12 have lower numbers of genes. (C) The repeat densities (percentage of the repeat-associated nucleotides) are different among different scaffolds.

Figure S3 | Identification of CentO repeats in $\boldsymbol{E}$. salsugineum. To infer the scaffold orientations, we searched for centromere-specific CentO satellite sequences that could be used to determine the centromeric ends of scaffolds. (A) A 177-bp motif (EsCentO) was found tandemly repeated over 500,000 times in E. salsugineum, sharing a similar AT-rich pattern with the 178-bp CentO repeats in A. thaliana. (B) The EsCentOs are abundant among the short scaffolds below $10 \mathrm{~kb}$ and often located at one terminus of a large scaffold, indicating the genome assembling was mostly interrupted at the edges of core centromeres. (C) Comparison of the CentO repeat motifs in $A$. thaliana and $E$. salsugineum shows the similar size and AT-rich feature.

Figure S4 | Comparison of the numbers of transcription factor families in the $\boldsymbol{A}$. thaliana and $\boldsymbol{E}$. salsugineum. Most TF families contain similar numbers of members between the two species.

Figure S5 | (A) GO enrichment analysis shows that the two species has similar number of genes in each functional category. The GO enrichment analysis was performed by AgriGO database. (B) The genes involved in ubiquitin related protein modification pathways are over represented in $E$. salsugineum than in $A$. thaliana in category of Biological Process. (C) Over represented GO classes in Cellular Components (Left) and Molecular Function (Right). Genes in the "ubiquitin-protein ligase activity" are overrepresented in E. salsugineum.

Miocene origin for Arabidopsis thaliana. Proc. Natl. Acad. Sci. U.S.A. 107, 18724-18728.

Bouchereau, A., Lugan, R., Niogret, M. F., Leport, L., Guegan, J. P., Larher, F. R., et al. (2010). Metabolome and water homeostasis analysis of Thellungiella salsuginea suggests that dehydration tolerance is a key response to osmotic stress in this halophyte. Plant J. 64, 215-229.

Bressan, R. A., Zhang, C. Q., Zhang, H., Hasegawa, P. M., Bohnert, H. J., and Zhu, J. K. (2001). Learning from the Arabidopsis experience. The next gene search paradigm. Plant Physiol. 127, 1354-1360.

Cheng, F. W. J., Fang, L., and Wang, X. (2012). Syntenic gene analysis between Brassica rapa and other Brassicaceae species. Front. Plant Sci. 3:198. doi:10.3389/fpls.2012.00198

Dassanayake, M., Oh, D. H., Haas, J. S., Hernandez, A., Hong, H., Ali, S., et al. (2011). The genome of the extremophile crucifer Thellungiella parvula. Nat. Genet. 43, 913-918. 
Deng, C., Cheng, C. H., Ye, H., He, X., and Chen, L. (2010). Evolution of an antifreeze protein by neofunctionalization under escape from adaptive conflict. Proc. Natl. Acad. Sci. U.S.A. 107, 21593-21598.

Dorantes-Acosta, A. E., SanchezHernandez, C. V., and ArteagaVazquez, M. A. (2012). Biotic stress in plants: life lessons from your parents and grandparents. Front. Genet. 3:256. doi:10.3389/fgene.2012.00256

dos Reis, M., Savva, R., and Wernisch, L. (2004). Solving the riddle of codon usage preferences: a test for translational selection. Nucleic Acids Res. 32, 5036-5044.

Eddy, S. R. (1998). Profile hidden Markov models. Bioinformatics 14, 755-763.

Fang, L., Cheng, F., Wu, J., and Wang, X. (2012). The impact of genome triplication on tandem gene evolution in Brassica rapa. Front. Plant Sci. 3:261. doi:10.3389/fpls.2012.00261

Fedoroff, N. (2000). Transposons and genome evolution in plants. Proc. Natl. Acad. Sci. U.S.A. 97, 7002-7007.

Ford, B. A., Ernest, J. R., and Gendall, A. R. (2012). Identification and characterization of orthologs of AtNHX5 and AtNHX6 in Brassica napus. Front. Plant Sci. 3:208. doi:10.3389/fpls.2012.00208

Gagne, J. M., Downes, B. P., Shiu, S. H., Durski, A. M., and Vierstra, R. D. (2002). The F-box subunit of the SCF E3 complex is encoded by a diverse superfamily of genes in Arabidopsis. Proc. Natl. Acad. Sci. U.S.A. 99, 11519-11524.

Gibeaut, D. M., Hulett, J., Cramer, G. R., and Seemann, J. R. (1997). Maximal biomass of Arabidopsis thaliana using a simple, lowmaintenance hydroponic method and favorable environmental conditions. Plant Physiol. 115, 317-319.

Godfray, H. C., Beddington, J. R., Crute, I. R., Haddad, L., Lawrence, D., Muir, J. F., et al. (2010). Food security: the challenge of feeding 9 billion people. Science 327, 812-818.

Gong, Q. Q., Li, P. H., Ma, S. S., Rupassara, S. I., and Bohnert, H. J. (2005). Salinity stress adaptation competence in the extremophile Thellungiella halophila in comparison with its relative Arabidopsis thaliana. Plant J. 44, 826-839.

Hatje, K., and Kollmar, M. (2012). A phylogenetic analysis of the brassicales clade based on an alignment-free sequence comparison method. Front. Plant Sci. 3:192. doi:10.3389/fpls.2012.00192
Hittinger, C. T., and Carroll, S. B. (2007). Gene duplication and the adaptive evolution of a classic genetic switch. Nature 449, 677-681.

Hu, T. T., Pattyn, P., Bakker, E. G., Cao, J., Cheng, J. F., Clark, R. M., et al. (2011). The Arabidopsis lyrata genome sequence and the basis of rapid genome size change. Nat. Genet. 43, 476-481.

Innan, H., and Kondrashov, F. (2010). The evolution of gene duplications: classifying and distinguishing between models. Nat. Rev. Genet. 11, 97-108.

Jiang, H., Zhang, Y., Sun, J., Wang, W., and $\mathrm{Gu}, \mathrm{Z}$. (2008). Differential selection on gene translation efficiency between the filamentous fungus Ashbya gossypii and yeasts. BMC Evol. Biol. 8:343. doi:10.1186/1471-21488-343

Kent, W. J. (2002). BLAT - the BLASTlike alignment tool. Genome Res. 12, 656-664.

Khan, I. A. (1982). Managing salinity in irrigated agriculture. J. Irrigat. Drain. Div. 108, 43-56.

Kim, J. H., Woo, H. R., Kim, J., Lim, P. O., Lee, I. C., Choi, S. H., et al. (2009). Trifurcate feed-forward regulation of age-dependent cell death involving miR164 in Arabidopsis. Science 323, 1053-1057

Krzywinski, M., Schein, J., Birol, I., Connors, J., Gascoyne, R., Horsman, D., et al. (2009). Circos: an information aesthetic for comparative genomics. Genome Res. 19, 1639-1645.

Kumekawa, N., Hosouchi, T., Tsuruoka, H., and Kotani, H. (2000). The size and sequence organization of the centromeric region of Arabidopsis thaliana chromosome 5. DNA Res. 7, 315-321.

Kumekawa, N., Hosouchi, T., Tsuruoka, H., and Kotani, H. (2001). The size and sequence organization of the centromeric region of Arabidopsis thaliana chromosome 4. DNA Res. 8, 285-290.

Langmead, B., Trapnell, C., Pop, M., and Salzberg, S. L. (2009). Ultrafast and memory-efficient alignment of short DNA sequences to the human genome. Genome Biol. 10, R25.

Lee, H. R., Neumann, P., MacAs, J., and Jiang, J. M. (2006). Transcription and evolutionary dynamics of the centromeric satellite repeat CentO in rice. Mol. Biol. Evol. 23, 2505-2520.

Li, L., Stoeckert, C. J. Jr., and Roos, D. S. (2003). OrthoMCL: identification of ortholog groups for eukaryotic genomes. Genome Res. 13, 2178-2189.
Li, W., Cui, X., Meng, Z., Huang, X., Xie, Q., Wu, H., et al. (2012). Transcriptional regulation of Arabidopsis MIR168a and argonautel homeostasis in abscisic acid and abiotic stress responses. Plant Physiol. 158, 1279-1292.

Lin, S. I., Chiang, S. F., Lin, W. Y., Chen, J. W., Tseng, C. Y., Wu, P. C., et al. (2008). Regulatory network of microRNA399 and PHO2 by systemic signaling. Plant Physiol. 147, 732-746.

Lowe, T. M., and Eddy, S. R. (1997). tRNAscan-SE: a program for improved detection of transfer RNA genes in genomic sequence. Nucleic Acids Res. 25, 955-964.

Luo, M., and Wing, R. A. (2003). An improved method for plant BAC library construction. Methods Mol. Biol. 236, 3-20.

Ma, C., Chen, H., Xin, M., Yang, R., and Wang, X. (2012). KGBassembler: a karyotype-based genome assembler for Brassicaceae species. Bioinformatics 28, 3141-3143.

Man, O., and Pilpel, Y. (2007). Differential translation efficiency of orthologous genes is involved in phenotypic divergence of yeast species. Nat. Genet. 39, 415-421.

Mandakova, T., and Lysak, M. A. (2008). Chromosomal phylogeny and karyotype evolution in $\mathrm{x}=7$ crucifer species (Brassicaceae). Plant Cell 20, 2559-2570.

Murashige, T., and Skoog, F. (1962). A revised medium for rapid growth and bio assays with tobacco tissue cultures. Physiol. Plant 15, 473-497.

Oh, D. H., Dassanayake, M., Haas, J. S., Kropornika, A., Wright, C., d'Urzo, M. P., et al. (2010). Genome structures and halophytespecific gene expression of the extremophile Thellungiella parvula in comparison with Thellungiella salsuginea (Thellungiella halophila) and Arabidopsis. Plant Physiol. 154, 1040-1052.

Oh, D. H., Leidi, E., Zhang, Q., Hwang, S. M., Li, Y., Quintero, F. J., et al. (2009). Loss of halophytism by interference with SOS1 expression. Plant Physiol. 151, 210-222.

Rhoades, M. W., Reinhart, B. J., Lim, L. P., Burge, C. B., Bartel, B., and Bartel, D. P. (2002). Prediction of plant microRNA targets. Cell 110, 513-520.

Saitou, N., and Nei, M. (1987). The neighbor-joining method: a new method for reconstructing phylogenetic trees. Mol. Biol. Evol. 4, 406-425.
Schranz, M. E., Lysak, M. A., and Mitchell-Olds, T. (2006). The ABC's of comparative genomics in the Brassicaceae: building blocks of crucifer genomes. Trends Plant Sci. 11, 535-542.

Simillion, C., Janssens, K., Sterck, L., and Van de Peer, Y. (2008). i-ADHoRe 2.0: an improved tool to detect degenerated genomic homology using genomic profiles. Bioinformatics 24 127-128.

Taji, T., Komatsu, K., Katori, T., Kawasaki, Y., Sakata, Y., Tanaka, S., et al. (2010). Comparative genomic analysis of 1047 completely sequenced cDNAs from an Arabidopsis-related model halophyte, Thellungiella halophila. BMC Plant Biol. 10:261. doi:10.1186/1471-2229-10-261

Taji, T., Seki, M., Satou, M., Sakurai, T., Kobayashi, M., Ishiyama, K., et al. (2004). Comparative genomics in salt tolerance between Arabidopsis and Arabidopsis-related halophyte salt cress using Arabidopsis microarray. Plant Physiol. 135, 1697-1709.

Tang, H., and Lyons, E. (2012). Unleashing the genome of Brassica rapa. Front. Plant Sci. 3:172. doi:10.3389/fpls.2012.00172

Thompson, J. D., Gibson, T. J., Plewniak, F., Jeanmougin, F., and Higgins, D. G. (1997). The CLUSTAL_X windows interface: flexible strategies for multiple sequence alignment aided by quality analysis tools. Nucleic Acids Res. 25, 4876-4882.

Wang, X., Wang, H., Wang, J., Sun, R., Wu, J., Liu, S., et al. (2011). The genome of the mesopolyploid crop species Brassica rapa. Nat. Genet. 43, 1035-1039.

Wessler, S. R. (1996). Turned on by stress. Plant retrotransposons. Curr. Biol. 6, 959-961.

Wong, C. E., Li, Y., Labbe, A., Guevara, D., Nuin, P., Whitty, B., et al. (2006). Transcriptional profiling implicates novel interactions between abiotic stress and hormonal responses in Thellungiella, a close relative of Arabidopsis. Plant Physiol. 140, 1437-1450.

Wright, S. I., and Agren, J. A. (2011). The Arabidopsis lyrata genome sequence Sizing up Arabidopsis genome evolution. Heredity 107, 509-510.

Wright, S. I., and Gaut, B. S. (2005). Molecular population genetics and the search for adaptive evolution in plants. Mol. Biol. Evol. 22, 506-519. 
Wu, H. J., Zhang, Z. H., Wang, J. Y., Oh, D. H., Dassanayake, M., Liu, B. H., et al. (2012). Insights into salt tolerance from the genome of Thellungiella salsuginea. Proc. Natl. Acad. Sci. U.S.A. 109, 12219-12224.

$\mathrm{Xu}, \mathrm{G}$., Ma, H., Nei, M., and Kong, H. (2009). Evolution of F-box genes in plants: different modes of sequence divergence and their relationships with functional diversification. Proc. Natl. Acad. Sci. U.S.A. 106, 835-840.

Yang, J., Osman, K., Iqbal, M., Stekel, D. J., Luo, Z., Armstrong, S. J., et al. (2012). Inferring the Brassica rapa interactome using protein-protein interaction data from Arabidopsis thaliana. Front. Plant Sci. 3:297. doi:10.3389/fpls.2012.00297

Yang, X., and Li, L. (2011). miRDeepP: a computational tool for analyzing the microRNA transcriptome in plants. Bioinformatics 27, 2614-2615.

Conflict of Interest Statement: The authors declare that the research was conducted in the absence of any commercial or financial relationships that could be construed as a potential conflict of interest.

Received: 22 January 2013; paper pending published: 15 February 2013; accepted: 24 February 2013; published online: 21 March 2013.

Citation: Yang R, Jarvis DE, Chen H, Beilstein MA, Grimwood J, Jenkins J, Shu S, Prochnik S, Xin M, Ma C, Schmutz J, Wing RA, Mitchell-Olds T, Schumaker KS and Wang $X$ (2013) The reference genome of the halophytic plant Eutrema salsugineum. Front. Plant Sci. 4:46. doi: 10.3389/fpls.2013.00046
This article was submitted to Frontiers in Plant Genetics and Genomics, a specialty of Frontiers in Plant Science.

Copyright () 2013 Yang, Jarvis, Chen, Beilstein, Grimwood, Jenkins, Shu, Prochnik, Xin, Ma, Schmutz, Wing, Mitchell-Olds, Schumaker and Wang. This is an open-access article distributed under the terms of the Creative Commons Attribution License, which permits use, distribution and reproduction in other forums, provided the original authors and source are credited and subject to any copyright notices concerning any third-party graphics etc. 